\title{
Infecciones por Staphylococcus aureus meticilino resistente adquirido en la comunidad: hospitalización y riesgo de letalidad en 10 centros pediátricos de Argentina Community-acquired methicillin-resistant Staphylococcus aureus infections: hospitalization and case fatality risk in 10 pediatric facilities in Argentina
}

a. Hospital de Niños Ricardo Gutiérrez, Ciudad Autónoma de Buenos Aires.

b. Hospital de Niños Víctor Vilela, Rosario, Santa Fe.

c. Hospital de Niños Pedro de Elizalde, Ciudad Autónoma de Buenos Aires.

d. Hospital de Niños de San Justo, Buenos Aires.

e. Hospital del Niño Jesús, San Miguel de Tucumán.

f. Hospital de Niños Dr. Héctor Quintana, Jujuy.

g. Hospital de Niños Eva Perón, San Fernando del Valle de Catamarca.

h. Hospital de Niños Orlando Alassia, Santa Fe.

i. Hospital Pediátrico Humberto Notti, Guaymallén, Mendoza.

j. Hospital Pediátrico J. Pablo II,

Corrientes.

Correspondencia: Dra. Ángela Gentile: angelagentile21@gmail. com

Financiamiento:

Ninguno que declarar.

Conflicto de intereses:

Ninguno que declarar.

Recibido: 21-3-2017

Aceptado: 29-8-2017

\author{
Dra. Ángela Gentile ${ }^{a}$ Dra. Julia Bakira , Dra. Gabriela Ensinck ${ }^{b}$, Dr. Aldo Cancellarac, \\ Dr. Enrique V. Casanueva ${ }^{d}$, Dra. Verónica Firpo ${ }^{e}$, Dr. Martín Carusof, \\ Dra. María F. Lución ${ }^{a}$, Dr. Alejandro Santillán Iturres ${ }^{g}$, Dra. Fabiana Molina ${ }^{h}$, \\ Dr. Héctor J. Abate $e^{i}$ Dra. Andrea Gajo Ganej, Dr. Santiago López Papuccib y \\ Grupo de Trabajo de Staphylococcus aureus*
}

\section{RESUMEN}

Introducción. Las infecciones por Staphylococcus aureus meticilino resistente adquirido en la comunidad (SAMR-C) son prevalentes en Argentina y el mundo; pueden tener evolución grave.

Objetivos: Estimar tasa de hospitalización y factores de riesgo de letalidad de la infección por SAMR-C.

Métodos. Estudio analítico transversal. Se incluyeron todos los pacientes $\leq 15$ años con infección por Staphylococcus aureus adquirido en la comunidad (SA-C) hospitalizados en 10 centros pediátricos, entre enero/2012-diciembre/ 2014. Resultados. Del total de 1141 pacientes con infección por SA-C, 904 (79,2\%) fueron SAMR-C. La tasa de hospitalización de casos de SAMR-C (por 10000 egresos) en < 5 años fue 27,6 en 2012, $35,2$ en 2013 y 42,7 en 2014 ( $p=0,0002)$. El grupo de 2-4 años fue el más afectado: 32,2, 49,4 y 54,4, respectivamente $(p=0,0057)$. Las presentaciones clínicas fueron infección de piel y partes blandas (IPPB): $66,2 \%$; neumonía: $11,5 \%$; sepsis / bacteriemia: $8,5 \%$; osteomielitis: 5,5\%; artritis: 5,2\%; absceso de psoas: $1,0 \%$; pericarditis/ La resistencia antibiótica fue, para eritromicina, trimetoprima-sulfametoxazol: $0,6 \%$. Todas las cepas fueron sensibles a vancomicina. La letalidad fue $2,2 \%$ y los factores de riesgo asociados fueron [OR (IC 95\%)] edad $\geq 8$ años $(2,78 ; 1,05-7,37)$, neumonía $(6,37 ; 2,37-17,09)$, meningitis $(19,53 ; 2,40-127,87)$ y sepsis/ bacteriemia $(39,65 ; 11,94-145,55)$.

Conclusiones. La tasa de infección por SAMR-C endocarditis: $0,8 \%$; meningitis: $0,6 \%$; otras: $0,7 \%$. $11,1 \%$; clindamicina, $11,0 \%$; gentamicina, $8,4 \%$; fue alta; la tasa de hospitalización aumentó en 2013-14; el grupo de 2-4 años fue el más afectado. Presentaron mayor riesgo de letalidad los $\geq 8$ años y las clínicas de neumonía, meningitis y sepsis. Palabras clave: infecciones estafilocócicas, letalidad, factores de riesgo, pediatría.

http:/ / dx.doi.org/10.5546/ aap.2018.e47 Texto completo en inglés:

http:/ / dx.doi.org/10.5546/aap.2018.eng.e47

Cómo citar: Gentile Á, Bakir J, Ensinck G, et al. Infecciones por Staphylococcus aureus meticilino resistente adquirido en la comunidad: hospitalización y riesgo de letalidad en 10 centros pediátricos de Argentina. Arch Argent Pediatr 2018;116(1):e47-e53.

\section{Glosario}

h: horas

IPPB: infección de piel y partes

blandas

OR: odds ratio

PCV13: vacuna neumocócica conjugada 13 valente PVL: leucocidina Panton-Valentine SA: Staphylococcus aureus SA-C: Staphylococcus aureus de la comunidad SAMR: Staphylococcus aureus meticilino resistentes
* Grupo de trabajo de Staphylococcus aureus: Lic. Dra. Nanci Miguez,

Dra. Ivana Morales, Dra. Laura Miño, Dra. Macarena Uranga,

Dra. Norma Fernández, Dra. María R. Miranda, Dra. Patricia Carrizo Herrera, Dr. Pablo Scoleri, Bioq. María R. Baroni y Dra. Carolina Aguirre. 
SAMR-C: Staphylococcus aureus meticilino resistente adquirido en la comunidad SAMR-H: Staphylococcus aureus meticilino resistentes de origen hospitalario

SAMS: Staphylococcus aureus meticilino sensibles TMP-SMZ: trimetoprima-sulfametoxazol

\section{INTRODUCCIÓN}

Staphylococcus aureus ( $S A$ ) es un microorganismo ampliamente distribuido en el medio. Coloniza la piel y las membranas mucosas del 30\% al 50\% de los niños y adultos sanos. Puede causar un amplio espectro de infecciones, desde las leves de la piel y tejidos blandos hasta las invasivas, como neumonía, bacteriemia y sepsis. ${ }^{1}$

Años después de la introducción de la meticilina, en 1959, se informaron brotes de infecciones por $S A$ meticilino resistentes (SAMR). ${ }^{2}$ Inicialmente, los casos de infección por SAMR eran de origen hospitalario (SAMR-H) o asociados a la atención médica. ${ }^{3}$

A partir de los noventa, se informaron casos de pacientes colonizados o infectados con cepas de SAMR adquiridas en la comunidad (SAMR-C) en distintas áreas de los Estados Unidos y el mundo. ${ }^{4,5}$ Los brotes documentados se registraron en comunidades con condiciones de hacinamiento, presidiarios, reclutas, niños en guarderías, deportistas de contacto, homosexuales $y$ adictos endovenosos. Presentaron, principalmente, infecciones de piel y partes blandas (IPPB) y algunos casos evolucionaron a enfermedad invasiva. ${ }^{6,7}$

En la Argentina, Paganini y cols., identificaron estas infecciones en un hospital pediátrico hacia el año $2004^{8}$ y luego informaron en un estudio multicéntrico tasas de SAMR-C que superaban el $60 \%$ en la mayoría de los centros. ${ }^{9}$ Otros trabajos nacionales más recientes, con diferentes metodologías, notificaron tasas del $55 \%$ en 66 hospitales de adultos y pediátricos, en noviembre de $2009,{ }^{10}$ y resistencia del $65 \%$ en infecciones bacteriémicas por $S A$ de la comunidad (SA-C). ${ }^{11}$

Las cepas SAMR-C mostraron un patrón de sensibilidad a los antibióticos diferente de las de origen hospitalario. Estas últimas eran, en general, multirresistentes, mientras que las cepas comunitarias presentaban solamente resistencia a la meticilina, con sensibilidad variable a eritromicina/clindamicina y alta sensibilidad a trimetoprima-sulfametoxazol (TMP-SMZ). ${ }^{4}$

$\mathrm{Si}$ bien los genes que codifican la resistencia a meticilina de las cepas hospitalarias y comunitarias son los mismos, el cassette cromosómico estafilocócico en el cual están integrados esos genes es diferente, lo que indica que las cepas de la comunidad no representan una diseminación de las cepas hospitalarias. ${ }^{12,13}$ Asimismo, una citotoxina llamada leucocidina Panton-Valentine (PVL) presente en las cepas SAMR-C y en algunas $S A$ meticilino sensibles (SAMS) se asoció a IPPB, ${ }^{4,14}$ invasividad, osteomielitis, neumonía necrotizante y mal pronóstico. ${ }^{4,14-18}$ Sin embargo, recientemente, se ha discutido su rol como determinante de virulencia. $^{19}$

Los objetivos del presente estudio fueron estimar la tasa de hospitalización de la infección por SAMR-C, describir las características clínico-epidemiológicas y microbiológicas de los pacientes, y analizar los factores de riesgo de letalidad en centros seleccionados que representaban diferentes áreas del país.

\section{POBLACIÓN Y MÉTODOS}

Diseño: Estudio epidemiológico observacional transversal, con captura prospectiva de datos.

Lugar de estudio: Participaron 10 centros pediátricos (según regiones).

a. Noroeste: Hospital del Niño Jesús (Tucumán), Hospital de Niños H. Quintana (Jujuy), Hospital de Niños E. Perón (Catamarca).

b. Nordeste: Hospital Pediátrico J. Pablo II (Corrientes).

c. Cuyo: Hospital Pediátrico H. Notti (Mendoza).

d. Centro: Hospital de Niños Víctor J. Vilela (Rosario), Hospital de Niños O. Alassia (Santa $\mathrm{Fe})$.

e. Buenos Aires: Hospital de Niños de San Justo (Bs. As.), Hospital de Niños P. de Elizalde (Ciudad Autónoma de Buenos Aires -CABA-), Hospital de Niños R. Gutiérrez (CABA).

Todos los centros contaban con recursos humanos, logísticos e infraestructura adecuada para la atención del paciente internado, realización de diagnóstico por imágenes y estudios bacteriológicos.

Criterios de inclusión: Se incluyeron todos los pacientes $\leq 15$ años con infección por SA-C, hospitalizados en los 10 centros pediátricos, entre enero de 2012 y diciembre de 2014 inclusive.

Los pacientes fueron ingresados a través de la vigilancia epidemiológica activa en las salas de internación. Posteriormente, se completó una ficha confeccionada para tal fin en la que constaban datos referentes al centro participante, código de identificación del paciente, fecha de 
ingreso, datos demográficos, variables clínicoepidemiológicas (edad, sexo, vacunación, número de personas en la casa, número de personas que dormían juntas, nivel socioeconómico, enfermedades subyacentes, estado nutricional, tabaquismo pasivo, internaciones previas en el último año, antibióticos previos en los últimos 3 meses), formas clínicas, cultivos bacteriológicos y susceptibilidad antibiótica, sitios de aislamiento, tratamiento antibiótico y evolución clínica (complicaciones y estado al egresar).

Además, se completó una planilla mensual de número de casos con infección por SA-C y SAMR-C, y número total de egresos hospitalarios.

\section{Definiciones}

Caso de infección por SA-C: Paciente que presentó aislamiento de $S A$ de un sitio normalmente estéril en forma ambulatoria, dentro de las primeras 48 horas (h) del ingreso al hospital o después de las $48 \mathrm{~h}$ de hospitalización, pero con clara evidencia clínica que sugería que la infección había sido adquirida en la comunidad [ej.: paciente sin internación previa que ingresaba con osteomielitis $\mathrm{y}$, después de $48 \mathrm{~h}$, se le realizaba drenaje quirúrgico, en cuya muestra desarrolló $S A]^{4,20}$

Caso de infección por $S A M R-C$ : Ídem caso a) en el cual se identificó SAMR.

Para definir el nivel socioeconómico, se utilizó el test de Graffar-Méndez Castellanos ${ }^{21}$ y se simplificó la clasificación en nivel alto (correspondiente al estrato I), medio (estratos II y III) y bajo (estratos IV y V).

Las enfermedades subyacentes incluyeron aquellas que comprometían la piel (tales como varicela, quemadura, traumatismo, dermatopatía, perforación, otras), enfermedades pulmonares crónicas (enfermedad pulmonar obstructiva crónica, fibrosis quística, enfisema y asma), enfermedades cardiovasculares (defectos congénitos del corazón, insuficiencia cardíaca congestiva, miocardiopatías, cardiopatía hipertensiva, hipertensión pulmonar, cardiopatía valvular, arritmias), enfermedades del hígado y del riñón, enfermedades metabólicas (como la diabetes), problemas neurológicos o del neurodesarrollo (parálisis cerebral, convulsiones, apoplejía, retraso mental, lesiones medulares o distrofias musculares), alteraciones hematológicas (anemia, asplenia funcional o anatómica), trastornos cromosómicos y genéticos, inmunosupresión debida a enfermedad o medicamentos (ej.: infección por virus de la inmunodeficiencia humana-VIH-, cáncer o corticoterapia a largo plazo) y déficit de complemento.
El diagnóstico del estado nutricional se efectuó según edad, peso y talla. Se siguieron los lineamientos de la Sociedad Argentina de Pediatría del año $2013^{22}$ para la definición de obesidad (puntaje $\mathrm{z}>2$ ) y desnutrición (puntaje $\mathrm{z}<2$ o un percentilo de peso para la edad $<3$ ).

Se consideró tabaquismo pasivo [criterio de la Organización Mundial de la Salud (OMS)] la exposición involuntaria al humo ambiental de tabaco (HAT) durante más de 15 minutos por día, más de 1 vez por semana.

$S A$ fue identificado mediante los métodos convencionales en los laboratorios de Bacteriología de los respectivos hospitales. Los antibiogramas se interpretaron según las normas del Clinical and Laboratory Standards Institute (CLSI) vigentes. ${ }^{23}$

\section{Consideraciones éticas}

Toda la información obtenida fue almacenada en una base de datos de acceso restringido. Los datos personales fueron registrados por medio de un código alfanumérico para que los investigadores estuvieran ciegos a la identificación del paciente.

El estudio contó con la aprobación de los Comités de Ética en Investigación de los hospitales respectivos.

\section{Análisis estadístico}

Los resultados fueron expresados en media \pm desvío estándar, mediana (rango) para la variable numérica edad. Las variables categóricas se expresaron en términos de porcentaje y se analizaron con el test $\chi^{2}$ con corrección de Yates.

Fueron identificados, inicialmente, los siguientes factores de riesgo potenciales de letalidad: variables clínico-epidemiológicas y formas clínicas, y la medida de asociación utilizada fue el odds ratio (OR), con un intervalo de confianza del 95\% (IC 95\%).

Finalmente, se realizó regresión logística para determinar los predictores independientes y se incluyeron, en forma manual, aquellas variables independientes que tuvieron una $\mathrm{p}=0,10$ o menor en el estudio univariado.

Se consideró como significativo un valor de $p$ menor de 0,05 .

La tasa de hospitalización de casos por SAMR-C anual se calculó dividiendo el total de casos de infección por SAMR-C por el total de egresos por año y por grupo etario, multiplicado por 10000 . 
Para el análisis descriptivo y univariado y para el cálculo del chi cuadrado de tendencia de las tasas de hospitalización, se utilizó el programa Epi Info versión 7 (CDC, Atlanta). Para el análisis multivariado, se utilizó el programa SPSS Statistics versión 17.0.

\section{RESULTADOS}

Sobre un total de 252050 pacientes internados en los 10 centros, $1141(0,45 \%)$ eran casos con infección por SA-C y, de estos, $904(79,2 \%)$ fueron SAMR-C. En las Tablas 1 y 2, se presentan las tasas de hospitalización anuales de casos de SA-C y SAMR-C por grupo etario, respectivamente.

Del total de 904 pacientes con infección por SAMR-C, la media de edad fue 63,7 meses (desvío estándar 55,5); la mediana, de 44,0 meses (rango $1-190) ; 33,5 \%$ eran < 2 años; y $61,5 \%$, de sexo masculino. El 51,4\% portaba carnet de vacunación, de los cuales 90,5\% tenía vacuna Haemophilus influenzae b y 66,3\% (134/202) de los < 24 meses tenía vacuna neumocócica conjugada 13 valente (PCV13). El 33,1\% era de nivel socioeconómico medio y el 65,3\%, bajo. En 830 casos, se consignó el número de personas en la casa, que fue $>5$ en el 49,6\%; asimismo, el número de personas en la habitación $>3$ fue del 37,2\% (294/790). E1 35,0\% (316/904) de los casos presentaba enfermedad de base (96 casos con más de una enfermedad), que, en orden de frecuencia, fueron las siguientes: la enfermedad respiratoria crónica
$(33,5 \%)$, enfermedad dermatológica $(20,9 \%)$, anemia $(19,7 \%)$, enfermedad neurológica $(8,3 \%)$, enfermedad cardiovascular $(2,9 \%)$, enfermedad genética $(1,7 \%)$, enfermedad renal/metabólica $(1,5 \%)$, enfermedad hepática $(0,5 \%)$, desnutrición $(4,3 \%)$, obesidad $(2,6 \%)$, inmunosupresión $(4,1 \%)$. El 4,2\% de los casos tenía antecedente de prematurez; $19,2 \%$, tabaquismo pasivo; $14,7 \%$, internaciones previas en el último año, y 7,7\%, tratamiento antibiótico previo en los últimos 3 meses.

Cuatro pacientes presentaron infecciones mixtas, 3 casos de SAMR-C y Haemophilus influenzae b y 1 caso de SAMR-C y Streptococcus $\beta$ hemolítico grupo A.

Las presentaciones clínicas se describen en la Tabla 3; presentaron formas clínicas combinadas 227 pacientes.

El 60,2\% (451/749) de las IPPB correspondían a pacientes $\sin$ factores predisponentes relacionados con los servicios de salud (enfermedad de base o antecedente de hospitalización en el último año). Asimismo, 6 de los 7 casos de meningitis también eran pacientes previamente sanos.

El diagnóstico microbiológico se realizó a partir de 1003 muestras: piel/ tejido blando $(67,4 \%)$, sangre $(16,8 \%)$, líquido pleural $(8,0 \%)$, líquido articular $(4,3 \%)$, hueso $(2,4 \%)$, líquido cefalorraquídeo (LCR) (0,6\%), otros (0,5\%). La resistencia antibiótica de las cepas de SAMR-C se describe en la Figura 1.

TABLA 1. Tasas de hospitalización de casos de infección por Staphylococcus aureus adquirido en la comunidad por grupo etario, en 10 centros hospitalarios, Argentina, 2012-2014

\begin{tabular}{lcccccccccc}
\hline Grupo etario & \multicolumn{3}{c}{$\mathbf{2 0 1 2}$} & \multicolumn{3}{c}{$\mathbf{2 0 1 3}$} & & $\mathbf{2 0 1 4}$ & \multicolumn{2}{c}{$\begin{array}{c}\text { Significación } \\
\text { estadística }\end{array}$} \\
\cline { 2 - 11 } & Egresos & $\mathbf{n}$ & Tasa por 10000 & Egresos & $\mathbf{n}$ & Tasa por 10000 & Egresos & n & Tasa por 10000 & $p$ \\
\hline 0-23 meses & 35979 & 112 & 31,1 & 32029 & 134 & 41,8 & 32787 & 145 & 44,2 & 0,0126 \\
24-59 meses & 17990 & 77 & 42,8 & 15987 & 97 & 60,7 & 15447 & 95 & 61,5 & 0,0291 \\
$<$ < años & 53969 & 189 & 35,0 & 48016 & 231 & 48,1 & 48234 & 240 & 49,8 & 0,0004 \\
5-15 años & 35980 & 128 & 35,6 & 32460 & 167 & 51,4 & 33391 & 186 & 55,7 & 0,0002 \\
\hline
\end{tabular}

TABla 2. Tasas de hospitalización de casos de infección por Staphylococcus aureus meticilino resistente adquirido en la comunidad por grupo etario, en 10 centros hospitalarios, Argentina, 2012-2014

\begin{tabular}{lcccccccccc}
\hline Grupo etario & \multicolumn{3}{c}{$\mathbf{2 0 1 2}$} & \multicolumn{3}{c}{$\mathbf{2 0 1 3}$} & & $\mathbf{2 0 1 4}$ & \multicolumn{2}{c}{$\begin{array}{r}\text { Significación } \\
\text { estadística }\end{array}$} \\
\cline { 2 - 11 } & Egresos & $\mathbf{n}$ & Tasa por 10000 & Egresos & $\mathbf{n}$ & Tasa por 10000 & Egresos & n & Tasa por 10000 & $p$ \\
\hline 0-23 meses & 35979 & 91 & 25,3 & 32029 & 90 & 28,1 & 32787 & 122 & 37,2 & 0,0127 \\
24-59 meses & 17990 & 58 & 32,2 & 15987 & 79 & 49,4 & 15447 & 84 & 54,4 & 0,0057 \\
$<$ 5 años & 53969 & 149 & 27,6 & 48016 & 169 & 35,2 & 48234 & 206 & 42,7 & 0,0002 \\
5-15 años & 35980 & 99 & 27,5 & 32460 & 125 & 38,5 & 33391 & 156 & 46,7 & 0,0001 \\
\hline
\end{tabular}


La tasa de letalidad global fue del 2,2\% $(20 / 904)$ y los factores de riesgo asociados fueron la edad $\geq 8$ años, las formas clínicas de neumonía, meningitis y sepsis/bacteriemia (Tabla 4). El predictor independiente de letalidad fue la manifestación clínica de sepsis / bacteriemia (OR 44,07; 12,07-160,87).

Las infecciones graves de tejidos blandos (fascitis y miositis) y las variables clínico-epidemiológicas, tales como sexo, vacunación, nivel socioeconómico, número de personas en la casa, número de personas que dormían juntas, enfermedad de base, prematurez, tabaquismo pasivo, internaciones previas en el último año y antibióticos previos en los últimos 3 meses, no se asociaron a mayor letalidad por infección por SAMR-C.

TABla 3. Formas clínicas de los casos de infección por Staphylococcus aureus meticilino resistente adquirido en la comunidad, en 10 centros hospitalarios, Argentina, 2012-2014

\begin{tabular}{lcc}
\hline Forma clínica & $\mathbf{N}$ & $\%$ \\
\hline Infección de piel y partes blandas* & 749 & 66,2 \\
Neumonía & 130 & 11,5 \\
Sepsis/bacteriemia & 97 & 8,5 \\
Osteomielitis & 62 & 5,5 \\
Artritis & 59 & 5,2 \\
Absceso del psoas & 11 & 1,0 \\
Pericarditis/endocarditis & 9 & 0,8 \\
Meningitis & 7 & 0,6 \\
Absceso renal & 2 & 0,2 \\
Absceso hepático & 2 & 0,2 \\
Peritonitis & 2 & 0,2 \\
Orquitis & 1 & 0,1 \\
\hline
\end{tabular}

* 27 casos de miositis y 4 casos de fascitis.

\section{DISCUSIÓN}

Las infecciones por SAMR-C son prevalentes en Argentina, así como en varios países del mundo ${ }^{4,5,8,9}$ y pueden tener evolución grave. ${ }^{15-18}$

En la actualidad, se reconoce que estas infecciones se producen en tres grupos específicos: las de origen hospitalario, las de la comunidad en pacientes con factores predisponentes relacionados con los servicios de salud y las de la comunidad en pacientes sin factores predisponentes. ${ }^{24,25}$ En el presente estudio, no se analizaron las primeras, pero sí las otras dos categorías en conjunto.

En nuestro estudio, la meticilino resistencia de la comunidad fue de casi el $80 \%$, mayor que la informada en los trabajos nacionales mencionados. Este aumento podría atribuirse a diferencias metodológicas en la inclusión de pacientes en los diferentes trabajos. ${ }^{8-11}$

Asimismo, se observó una tasa de hospitalización creciente de infección por SAMR-C a través de los 3 años de estudio.

TABLA 4. Factores de riesgo asociados a letalidad por infección por Staphylococcus aureus meticilino resistente adquirido en la comunidad, en 10 centros hospitalarios, Argentina, 2012-2014

\begin{tabular}{lccc}
\hline Factor de riesgo & \multicolumn{2}{c}{ Letalidad } & OR (IC 95\%) \\
& $\mathbf{N}$ & $\%$ & \\
\hline Sepsis/bacteriemia & $16 / 97$ & 16,5 & $39,65(11,94-145,55)$ \\
Meningitis & $2 / 7$ & 28,6 & $19,53(2,40-127,87)$ \\
Neumonía & $10 / 130$ & 7,7 & $6,37(2,37-17,09)$ \\
Edad $\geq 8$ años & $10 / 244$ & 4,1 & $2,78(1,05-7,37)$ \\
\hline
\end{tabular}

OR: odds ratio; IC: intervalo de confianza.

FIGURA 1. Resistencia antibiótica de las cepas de Staphylococcus aureus meticilino resistente adquirido en la comunidad en 10 centros hospitalarios, Argentina, 2012-2014

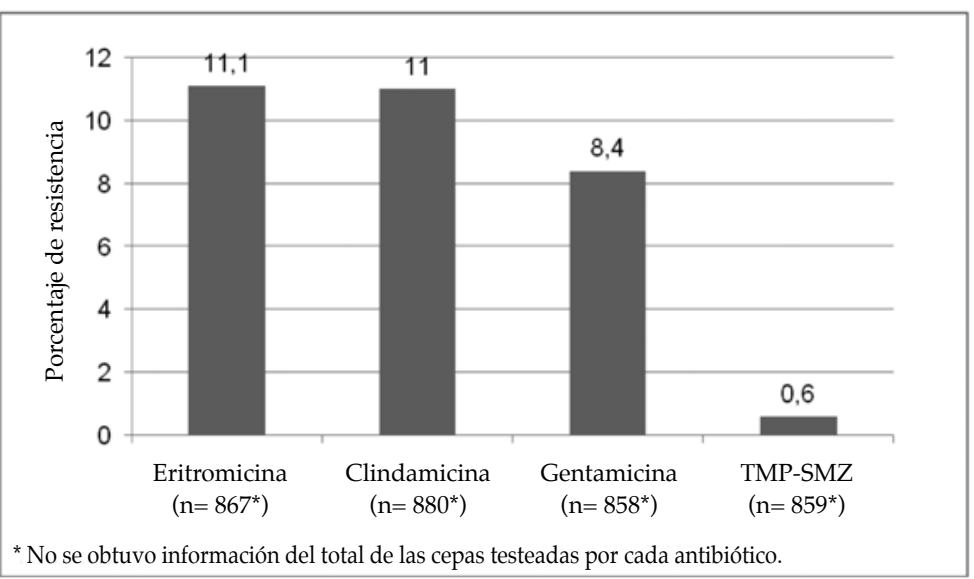


Estos hallazgos podrían explicarse por un fenómeno fundamentalmente ecológico: la colonización nasal con $S A$ aumentaría en los niños que han sido inmunizados con la vacuna neumocócica conjugada, probablemente, debido a la competencia entre esta vacuna y $S A .^{26,27}$ Sin embargo, en nuestro estudio, solo el $66 \%$ de los $<24$ meses tenía esquema completo para la PCV13. Además, las mayores tasas de hospitalización de infección por SAMR-C se evidenciaron en los niños escolarizados, que, generalmente, eran descritos como los más afectados $^{28}$ y los cuales no habían recibido PCV13 de Calendario. ${ }^{29}$

También se informó la relación de la colonización nasal con $S A$ y el tratamiento con amoxicilina-clavulanato en los tres meses anteriores. ${ }^{30}$ Nuestros datos muestran que un $7,7 \%$ de pacientes tenía antecedente de tratamiento antibiótico en los últimos 3 meses.

La infección por SAMR-C afectó, principalmente, los niveles sociales más pobres $(65 \%)$ y en condiciones de hacinamiento $(50 \%)$, como lo descrito en la literatura. ${ }^{28}$ Numerosos estudios consideran también como factor de riesgo para la adquisición de infecciones por $S A$ la colonización previa del paciente o de sus convivientes por dicho microorganismo en diferentes sitios de la superficie corporal, ${ }^{31}$ lo cual se vería favorecido en condiciones de hacinamiento.

El $42 \%$ de los pacientes con infección por SAMR-C tenía factores predisponentes relacionados con los cuidados de la salud, como internaciones previas en el último año $(15 \%)$ y/o alguna enfermedad subyacente (35\%). De estas últimas, las afecciones respiratorias fueron las más frecuentes, como ocurre en la población pediátrica en general, seguidas por afecciones dermatológicas, como varicela, quemaduras, picaduras, etc. Sin duda, las afecciones de la piel que interrumpen la integridad de la barrera cutánea favorecen la colonización y las infecciones por $S A \cdot, 31$

El tabaquismo pasivo se observó en casi el 20\% de los casos, lo que podría considerarse como un factor de riesgo de la enfermedad subyacente más frecuente de la población estudiada, la afección respiratoria. ${ }^{32}$

La infección por SAMR-C predominó en niños sanos, en quienes representó casi el 60\% de los episodios, y la forma clínica de presentación más frecuente fue la IPPB. Siguieron en frecuencia las enfermedades invasivas, tales como neumonía, sepsis/bateriemia e infecciones osteoarticulares. Dichas localizaciones en orden de frecuencia son coincidentes con lo publicado por diferentes autores, tanto a nivel nacional como internacional, en las que IPPB representan entre el $60 \%$ y el $90 \%$ de estos cuadros infecciosos. ${ }^{4,20,25}$ Hubo 4 casos con infecciones mixtas que compartieron la etiología con Haemophilus influenzae b y Streptococcus $\beta$ hemolítico grupo A.

Las publicaciones nacionales precedentes informan tasas de susceptibilidad antibiótica variables para las cepas SAMR-C. ${ }^{8-11,25}$ En nuestro estudio, si bien se incluyeron pacientes con infección por SAMR-C con factores predisponentes asociados a los cuidados de salud, el nivel de susceptibilidad a clindamicina y TMP-SMZ fue aceptable; además, todas las cepas fueron sensibles a vancomicina. Estos datos orientan al pediatra para la implementación de los tratamientos empíricos iniciales en las diferentes formas clínicas de presentación; clindamicina y TMP-SMZ continúan siendo antibióticos útiles, sobre todo, para el tratamiento de los pacientes con IPPB, y vancomicina se reserva para los niños con infecciones por SAMR-C graves, tales como bacteriemia, sepsis y endocarditis.

La letalidad del $2 \%$ fue semejante a la comunicada en las publicaciones nacionales citadas. Asimismo, los factores de riesgo asociados a letalidad, como la edad $\geq 8$ años, las formas clínicas de sepsis/bacteriemia, meningitis y neumonía, fueron similares a lo informado por otros estudios. . $^{433-35}$

Cabe destacar que la presencia de factores predisponentes, el sexo y las infecciones graves de tejidos blandos (fascitis y miositis) no se asociaron a mayor letalidad.

Por una parte, este trabajo tiene la fortaleza de todos los estudios multicéntricos, ser lo más aproximados posible a la realidad del país, ya que contemplan hospitales de referencia que atienden patología de alta, intermedia y baja complejidad, de distintas regiones. Por otra parte, tiene una debilidad inherente al tipo de estudio, ya que no todas las regiones están representadas. $Y$, si bien los centros trabajan con igual metodología, no están exentos de diferencias institucionales que podrían, en algunos casos, sesgar algunas de las variables estudiadas, especialmente, clínicas o microbiológicas.

\section{CONCLUSIONES}

La tasa de infecciones causadas por SAMR-C en niños fue alta; el foco clínico prevalente fue 
la IPPB y la mayor tasa de hospitalización se observó en los niños de 2-4 años.

La resistencia a clindamicina no superó el 15\%, por lo que se puede utilizar ante la sospecha de estas infecciones en niños.

La letalidad fue del $2 \%$. Fueron de mayor riesgo la edad $\geq 8$ años y las formas clínicas de neumonía, meningitis y sepsis.

\section{REFERENCIAS}

1. American Academy of Pediatrics. Staphylococcal Infections. En Kimberlin DW, Brady MT, Jackson MA, Long SS, eds. Red Book. 2015 Report of the Committee on Infectious Diseases 30th ed. Elk Grove Village, IL: American Academy of Pediatrics; 2015.Págs.715-32.

2. Benner EJ, Kayser FH. Growing clinical significance of methicillin-resistant Staphylococcus aureus. Lancet. 1968;2(7571):741-4.

3. Thompson RL, Cabezudo I, Wenzel RP. Epidemiology of nosocomial infections caused by methicillin-resistant Staphylococcus aureus. Ann Intern Med 1982;97(3):309-17.

4. Kaplan SL, Hulten KG, González BE, et al. Three-year surveillance of community-acquired Staphylococcus aureus infections in children. Clin Infect Dis 2005;40(12):1785-91.

5. Galiana Villar A. Infección por Staphylococcus aureus meticilino resistente adquirido en la comunidad. Arch Pediatr Urug 2003;74(1):26-9.

6. Centers for Disease Control and Prevention. Outbreaks of community-associated methicillin-resistant Staphylococcus aureus skin infections. Los Angeles County, California 2002-2003. MMWR Morb Mortal Wkly Rep. 2003;52(5):88.

7. Shahin R, Johnson I, Jamieson F, et al. Methicillin-resistant Staphylococcus aureus carriage in a child care center following a case of disease. Arch Pediatr Adolesc Med 1999;153(8):864-8.

8. Paganini $H$, Verdaguer V, Rodríguez AC, et al. Infecciones causadas por Staphylococcus aureus resistentes a la meticilina en niños provenientes de la comunidad en niños de la Argentina. Arch Argent Pediatr 2006;104(4):295-300.

9. Paganini H, Della Latta MP, Muller Opet B, et al. Estudio multicéntrico sobre las infecciones pediátricas por Staphylococcus aureus meticilino-resistente provenientes de la comunidad en la Argentina. Arch Argent Pediatr 2008;106(5):397-403.

10. Egea AL, Gagetti P, Lamberghini R, et al. New patterns of methicillin-resistant Staphylococcus aureus (MRSA) clones, community-associated MRSA genotypes behave like healthcare-associated MRSA genotypes within hospitals, Argentina. Int J Med Microbiol 2014;304(8):1086-99.

11. Pérez G, Martiren S, Reijtman V, et al. Bacteriemia por Staphylococcus aureus adquirido en la comunidad en niños: estudio de cohorte 2010-2014. Arch Argent Pediatr 2016;114(6):508-13.

12. Berger-Bächi B. Expression of resistance to methicillin. Trends Microbiol. 1994;2(10):389-93.

13. Buckingham SC, McDougal LK, Cathey LD, et al. Emergence of community-associated methicillin-resistant Staphylococcus aureus at a Memphis, Tennessee Children's Hospital. Pediatr Infect Dis J 2004;23(4):619-24.

14. Boubaker K, Diebold P, Blanc DS, et al. Panton-valentine leukocidin and staphyloccoccal skin infections in schoolchildren. Emerg Infect Dis 2004;10(1):121-4.

15. McCaskill ML, Mason EO Jr, Kaplan SL, et al. Increase of the USA300 clone among community-acquired methicillinsusceptible Staphylococcus aureus causing invasive infections. Pediatr Infect Dis J 2007;26(12):1122-7.

16. Martínez-Aguilar G, Avalos-Mishaan A, Hulten K, et al. Community-acquired, methicillin-resistant and methicillinsusceptible Staphylococcus aureus musculoskeletal infections in children. Pediatr Infect Dis J 2004;23(8):701-6.
17. Bocchini CE, Hulten KG, Mason EO Jr, et al. PantonValentine leukocidin genes are associated with enhanced inflammatory response and local disease in acute hematogenous Staphylococcus aureus osteomyelitis in children. Pediatrics 2006;117(2):433-40.

18. Gillet $Y$, Vanhems $P$, Lina G, et al. Factors predicting mortality in necrotizing community-acquired pneumonia caused by Staphylococcus aureus containing PantonValentine leukocidin. Clin Infect Dis 2007;45(3):315-21.

19. Shallcross LJ, Fragaszy E, Johnson AM, et al. The role of the Panton-Valentine leucocidin toxin in staphylococcal disease: a systematic review and meta-analysis. Lancet Infect Dis 2013;13(1):43-54.

20. Comité Nacional de Infectología. Infecciones de piel y partes blandas en pediatría: consenso sobre diagnóstico y tratamiento. Arch Argent Pediatr 2014;112(1):e96-102.

21. Méndez Castellano H, Méndez MC. Sociedad y Estratificación: Método Graffar-Méndez Castellano. Caracas: FUNDACREDESA; 1994.

22. Comité Nacional de Crecimiento y Desarrollo. Guía para la evaluación del crecimiento físico. 3. ${ }^{\mathrm{a}}$ ed. Buenos Aires: Sociedad Argentina de Pediatría; 2013.

23. Clinical and Laboratory Standards Institute. Performance Standards for AntimicrobialSusceptibility Testing; TwentyFive Informational Supplement (M100-S25). Wayne: CLSI; 2015. [Acceso: 29 de agosto de 2017]. Disponible en: http: / / www.facm.ucl.ac.be/intranet/CLSI/CLSI-2015-M100S25-original.pdf.

24. Harris A. Where did MRSA come from? [Consulta: 27 de octubre de 2016]. Disponible en: http:/ / www.uptodate. com/ contents / methicillin-resistant-staphylococcusaureus-mrsa-beyond-the-basics.

25. Paganini H, Della MP, Muller B, et al. Infecciones por Staphylococcus aureus resistente a meticilina adquirida en la comunidad en niños antes sanos y en niños relacionados al hospital en la Argentina. Rev Chilena Infectol 2009;26(5): 406-12.

26. Bogaert D, van Belkum A, Sluijter M, et al. Colonisation by Streptococcus pneumoniae and Staphylococcus aureus in healthy children. Lancet 2004;363(9424):1871-2.

27. Regev-Yochay G, DaganR, RazM, etal. Association between carriage of Streptococcus pneumoniae and Staphylococcus aureus in Children. JAMA. 2004;292(6):716-20.

28. Marcó del Pont J. Staphylococcus aureus de la comunidad, un problema reciente. Arch Argent Pediatr. 2016;114(6):500-1.

29. WHO vaccine-preventable diseases: monitoring system. 2016 global summary. Coverage time series for Argentina. [Acceso: 6 de abril de 2017]. Disponible en: http:/ / apps. who.int/immunization_monitoring/globalsummary/ coverages? $\mathrm{c}=\mathrm{ARG}$.

30. Guillemot D, Bonacorsi S, Blanchard JS, et al. Amoxicillinclavulanate therapy increases childhood nasal colonization by methicillin-susceptible Staphylococcus aureus strains producing high levels of penicillinase. Antimicrob Agents Chemother 2004;48(12):4618-23.

31. Lawrence H, Nopper A. Skin and Soft-Tissue Infections. En: Long S, Pickering L, Prober C, eds. Principles and Practice of Pediatric Infectious Disease. 4th ed. Philadelphia: Churchill Livingstone/Elsevier; 2012:427-35.

32. Martín Martín R, Sánchez Bayle M. Tabaquismo pasivo en la infancia y patología respiratoria de las vías bajas en una consulta de Atención Primaria. Rev Pediatr Aten Primaria 2009;11(44):587-95.

33. Fernández S, de Vedia L, López Furst MJ, et al. Methicillinresistant Staphylococcus aureus ST30-SCCmec IVc clone as the major cause of community-acquired invasive infections in Argentina. Infect Gen Evol 2013; 14:401-5.

34. Cobos-Carrascosa E, Soler-Palacin P, Nieves Larrosa M, et al. Staphylococcus aureus bacteremia in children: changes during eighteen years. Pediatr Infect Dis J 2015;34(12):1329-34.

35. Gonzalez BE, Martinez-Aguilar G, Hulten KG, et al. Severe Staphylococcal sepsis in adolescents in the era of community-acquired methicillin-resistant Staphylococcus aureus. Pediatrics. 2005;115(3):642-8 